Proceedings of the 16th Czech and Slovak Conference on Magnetism, Košice, Slovakia, June 13-17, 2016

\title{
Structural and Physical Properties of the new Stannide $\mathrm{Yb}_{3} \mathrm{Pd}_{4} \mathrm{Sn}_{13}$
}

\author{
F. Gastaldo ${ }^{a}$, M. Giovannini $^{a, b *}$, A. $\operatorname{Strydom}^{c}$, R.F. Djoumessi ${ }^{c}$, I. Čurlík $^{d}$, \\ M. ReIfFERS ${ }^{d}$, P. SOLOKHA ${ }^{a}$ AND A. SACCONE ${ }^{a}$ \\ ${ }^{a}$ Department of Chemistry, University of Genova, Via Dodecaneso 31, 15046, Genova, Italy \\ ${ }^{b}$ CNR-SPIN, Corso Ferdinando Maria Perrone 24, 16152, Genova, Italy \\ ${ }^{c}$ Highly Correlated Matter Research Group, Department of Physics, University of Johannesburg, South Africa \\ ${ }^{d}$ Faculty of Humanities and Natural Sciences, University of Prešov, Prešov, Slovakia
}

\begin{abstract}
Among the new discovered intermetallics in the $\mathrm{Yb}-\mathrm{Pd}-\mathrm{Sn}$ system, the cubic phase $\mathrm{Yb}_{3} \mathrm{Pd}_{4} \mathrm{Sn}_{13}$, with a lattice parameter of $0.9743(5) \mathrm{nm}$, emerges as a new member of the $\mathrm{R}_{3} \mathrm{~T}_{4} \mathrm{Sn}_{13}$ family $(\mathrm{R}=$ rare earth element, $\mathrm{T}=$ transition metal) crystallizing in the $\mathrm{Yb}_{3} \mathrm{Rh}_{4} \mathrm{Sn}_{13}$-structure type. The effective magnetic moment $\mu_{\text {eff }}=$ $1.84 \mu_{\mathrm{B}} / \mathrm{Yb}$ is strongly reduced. Moreover, measurements of magnetic properties, specific heat and resistivity indicate superconductivity below $2.4 \mathrm{~K}$. This compound is found to exhibit a strongly enhanced electronic specific heat at low temperature from which we infer a possible low-lying magnetic phase transition or other source of magnetic entropy.
\end{abstract}

DOI: 10.12693/APhysPolA.131.1006

PACS/topics: 71.27.+a, 74.25.F-, 74.10.+v

\section{Introduction}

Among the many materials types to which exploratory studies in condensed matter physics are being devoted, crystal structural classes such as the Heusler phases [1], cuprates [2], skutterudites [3] and others have received much prominence due to the useful classifying scheme that this approach offers. From a physical property point of view however, there is a preference to understand physical, magnetic and electronic properties in terms of physical phenomena such as the correlated electron class of materials, the heavy fermion ground state, unconventional superconductivity, and quantum criticality. The chemical element cerium is the most prominent element among systems hosting highly correlated electrons. While the correlated electron physics of ytterbium compounds are no less exciting, see for example [4] and references therein, there are much fewer Yb-based compounds known in this class. It is recognized that in $\mathrm{Yb}$ compounds the energy scales produced by hybridization of electrons of the $4 f$ orbital with conduction band electrons occurs on a much lower energy scale. In this work we report on one of the new compounds, $\mathrm{Yb}_{3} \mathrm{Pd}_{4} \mathrm{Sn}_{13}$, found as part of our research into the $\mathrm{Yb}-\mathrm{Pd}-\mathrm{Sn}$ ternary system [5] exhibiting very interesting compounds such as $\mathrm{Yb}_{2} \mathrm{Pd}_{2} \mathrm{Sn}[6]$ and $\mathrm{YbPd}_{2} \mathrm{Sn}[7]$.

\section{Synthesis and experimental methods}

The elements $\mathrm{Yb} \quad(99.9$ mass\% $\quad$ purity $), \quad \mathrm{Pd}$ (99.95 mass\% purity) and Sn (99.999 mass\% purity) were sealed in stoichiometric amount in tantalum

*corresponding author; e-mail: mauro.giovannini@unige.it crucibles. Crucibles were enclosed in a evacuated quartz vial and placed in a resistance furnace. A thermal cycle was then applied, consisting of a heating from room temperature to $600^{\circ} \mathrm{C}$ and slow cooling-down temperature ramp in order to improve the quality of the sample. The microstructure of the samples was investigated by optical and scanning electron microscopy and by quantitative electron probe microanalysis (SEMEPMA). X-ray diffraction (XRD) was performed on powder samples using the vertical diffractometer X'Pert MPD (Philips, Almelo, The Netherlands) with $\mathrm{Cu} K_{\alpha}$ radiation. The XRD patterns from the samples were processed using the POWDER CELL [8] program. Solid pieces of the synthesized compound $\mathrm{Yb}_{3} \mathrm{Pd}_{4} \mathrm{Sn}_{13}$ were prepared in suitable sizes and geometries for the experiments to be conducted. Magnetic properties were measured with a Squid-type magnetometer from Quantum Design San Diego, and physical properties with a Physical Properties Measurement System from the same manufacturers.

\section{Results and discussion}

The XRD patterns of the samples were successfully indexed on the basis of cubic $\mathrm{Yb}_{3} \mathrm{Rh}_{4} \mathrm{Sn}_{13}$ structure type with a lattice parameter of $0.9743(5) \mathrm{nm}$. A few weak peaks of $\mathrm{PdSn}_{2}$ (phase detected also by SEM) were always present as secondary phase. In the unit cell of the $\mathrm{Yb}_{3} \mathrm{Pd}_{4} \mathrm{Sn}_{13}$ compound (not shown) there are 40 atoms, but only one site for the magnetic $f$-electron element $\mathrm{Yb}$. Among the systems studied in our search for correlated cage compounds $[9,10]$, the $3: 4: 13$ series of compounds have received considerable attention as candidates for favorable or unusual thermoelectric properties [11]. Figure 1 shows the behaviour of dc-magnetic inverse susceptibility $1 / \chi(T)$ as function of temperature. The solid line 
is a fit of the Curie-Weiss law and demonstrates clear paramagnetic and stable-moment behavior over the temperature range $400-70 \mathrm{~K}$, according to a Weiss constant $\Theta_{p}=-30 \mathrm{~K}$ and an effective moment $\mu_{\mathrm{eff}}=1.84 \mu_{\mathrm{B}}$. The latter value is, however, less than half of what is expected from the full free-ion $\mathrm{Yb}^{3+}$ magnetic moment. In the inset of Fig. 1 the magnetic isotherms are plotted for two different temperatures. Compared to the regular paramagnetic behavior found at $T=20 \mathrm{~K}$, the $4.5 \mathrm{~K}$ magnetization by contrast suffers a strong curvature with field. Part (a) of Fig. 2 plots the progression of magnetization against applied fields collected at $1.8 \mathrm{~K}$.

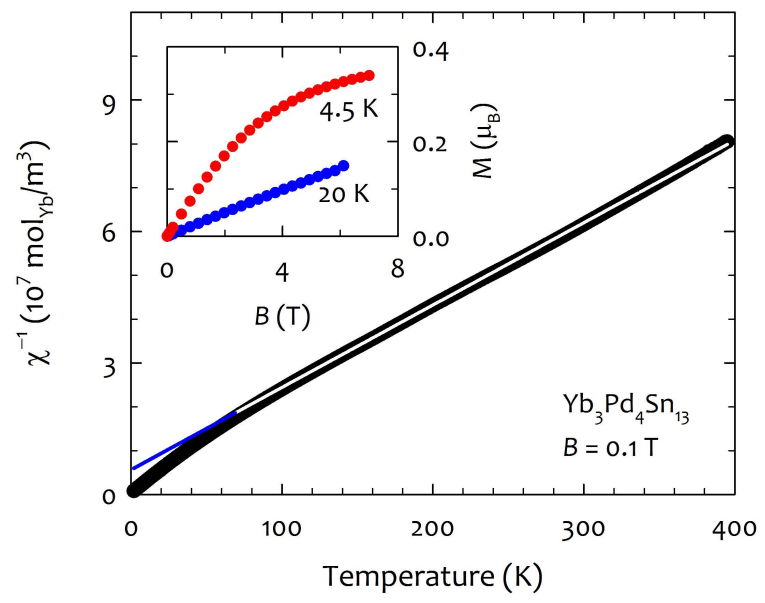

Fig. 1. (main part) Magnetic susceptibility against temperature, and (inset) magnetization against field at two fixed temperatures of $\mathrm{Yb}_{3} \mathrm{Pd}_{4} \mathrm{Sn}_{13}$.

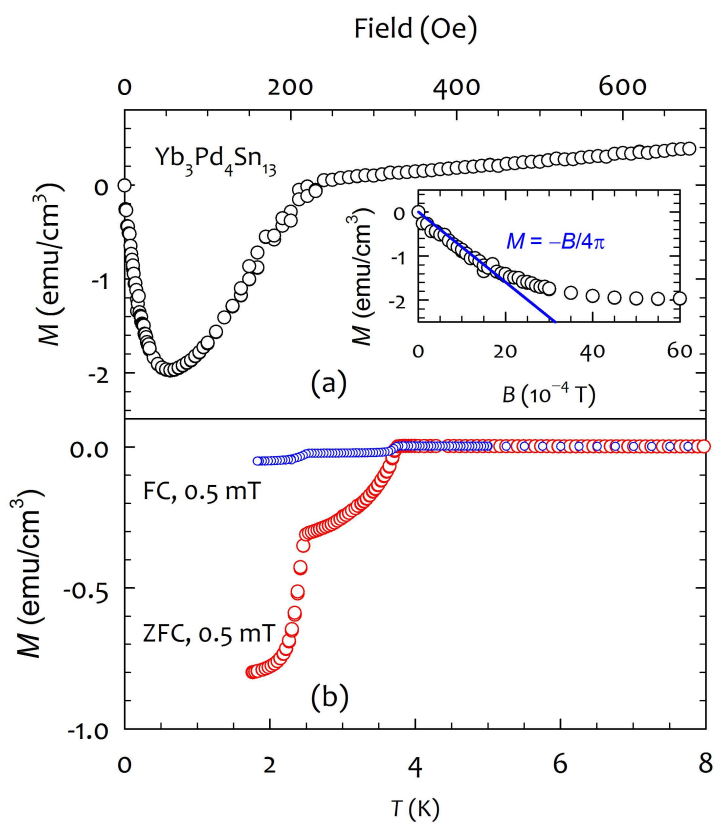

Fig. 2. (a) Magnetization vs. field at $T=1.8 \mathrm{~K}$, after cooling the sample in zero field. Inset: data at very low fields. (b) Magnetization vs. temperature in two different cooling protocols.
The inset shows that there is essentially complete screening of the sample, which is evidence for superconductivity of all or part of the sample. In part (b) this aspect is investigated through field-cooling susceptibility (in a dc field of $0.5 \mathrm{mT}$ ) which is the Meissner effect or flux expulsion, and field heating after zero-field cooling susceptibility, which is a demonstration of flux shielding. The Meissner effect in our experiment is relatively small, but one has to bear in mind that when there is sizeable flux pinning taking place in the mixed state a type II superconductor will not reveal a large flux expulsion. The temperature scans are in evidence of a double transition structure which we discuss further below. The electrical resistivity of $\mathrm{Yb}_{3} \mathrm{Pd}_{4} \mathrm{Sn}_{13}$ is shown in Fig. 3 against temperature (a) and field (b). The upper one of the two steps at $T_{\mathrm{C}}$ is eliminated already in very small fields (see specific heat further below) whereas the lower one of the two steps persists up to more than $0.5 \mathrm{~T}$. The highertemperature region demonstrates normal-metal behavior in $\rho(T)$ - although with a pronounced downward curvature.

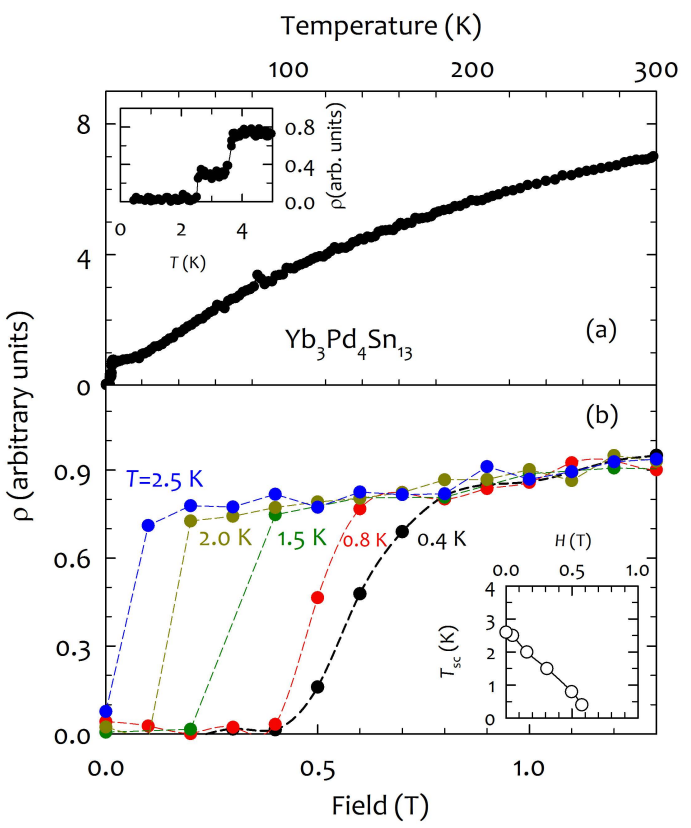

Fig. 3. (a) Electrical resistivity $\rho(T)$ below room temperature. Inset: the temperature region of the superconducting transition, with a two-step decrease to $\rho=0$. (b) $\rho$ vs. applied field at various temperatures. Inset: field dependence of the lower one of the two transitions seen in the inset of (a).

The superconducting transition is also evident in the specific heat - see Fig. 4. Here we show that $0.03 \mathrm{~T}$ is sufficient to quench the upper one of the two transitions, whereas the lower transition disappears between $0.5 \mathrm{~T}$ and $1.0 \mathrm{~T}$ as was also found in the resistivity of Fig. 4. With the jump measuring $\Delta C_{p}=120 \mathrm{~mJ} /(\mathrm{mol} \mathrm{K})$ the anomaly at $T_{\mathrm{C}}$ is small, but this has to be seen against the very high and strongly temperature dependent background of heat capacity, which is shown in Fig. 5. 


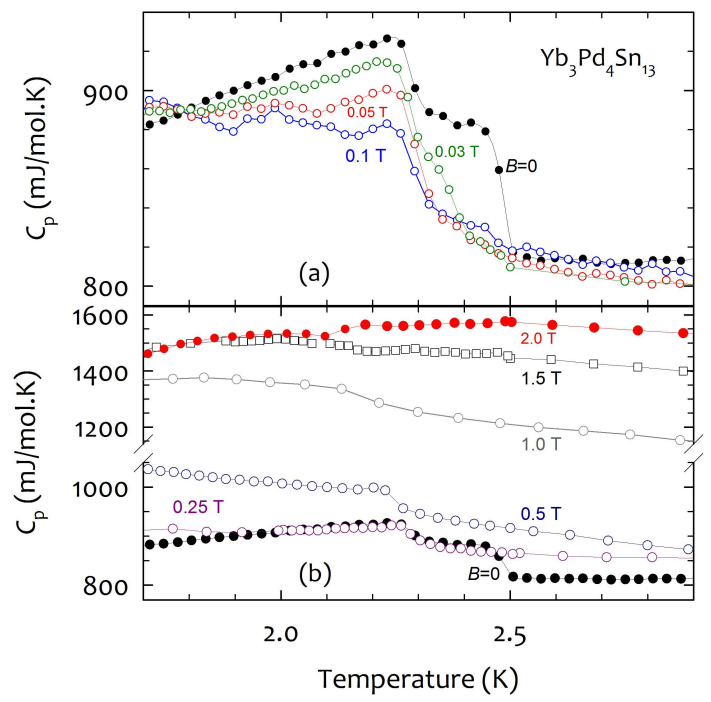

Fig. 4. Specific heat $C_{p}(T)$ of $\mathrm{Yb}_{3} \mathrm{Pd}_{4} \mathrm{Sn}_{13}$ for various low applied field values (a) and higher field (b). The transition disappears at $1.5 \mathrm{~T}$.

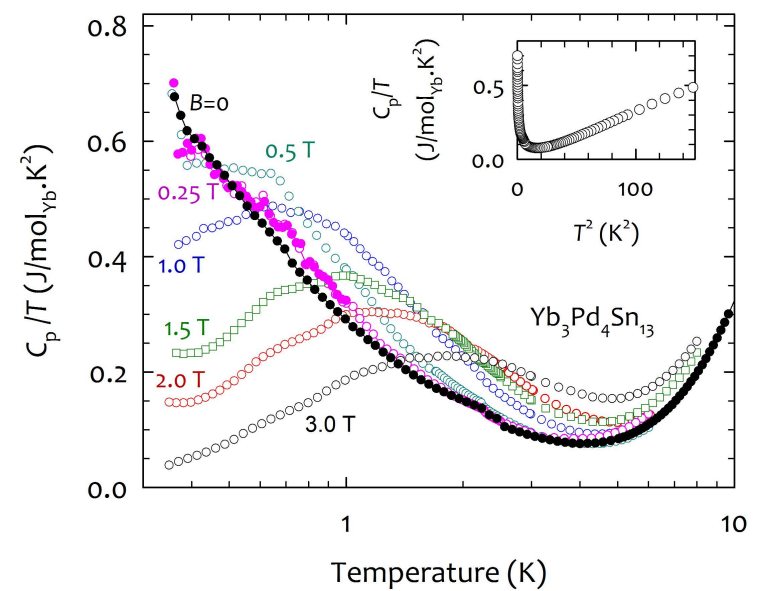

Fig. 5. Semi-log plot of specific heat $C_{p}(T)$ per mole $\mathrm{Yb}$ of $\mathrm{Yb}_{3} \mathrm{Pd}_{4} \mathrm{Sn}_{13}$ against temperature for various applied magnetic field values.

Compared to the jump in $C_{p}$ at $T_{\mathrm{C}}$, the zero-field electronic specific heat, associated with the specific heat in the form $C_{p}(T) / T$ rises dramatically by nearly an order of magnitude upon cooling below $4 \mathrm{~K}$. The specific heat of pure ytterbium has been reported as $1.305 \mathrm{~mJ} /(\mathrm{mol} \mathrm{K})$ at $0.3 \mathrm{~K}$ [12], of which $0.122 \mathrm{~mJ} /(\mathrm{mol} \mathrm{K})$ may be ascribable to the nuclear specific heat originating from nuclear hyperfine interactions. However, these contributions are negligible compared the total measured Yb-molar specific heat of $\mathrm{Yb}_{3} \mathrm{Pd}_{4} \mathrm{Sn}_{13}$ over the entire range plotted in Fig. 5, and we are thus concluding that the rise in $C_{p}(T) / T$ possibly belongs to the entropy of a very lowlying phase transition.

\section{Summary}

Our exploratory studies on the new member $\mathrm{Yb}_{3} \mathrm{Pd}_{4} \mathrm{Sn}_{13}$ of the cubic 3:4:13 family have revealed that this is a magnetic compound with electrical resistivity of a metallic nature. A pair of closely-spaced superconducting transitions are found below $2.5 \mathrm{~K}$. From the evolution of the specific heat in fields we cannot exclude that the upper of the two transitions may originate from free $\mathrm{Sn}$ in the sample. It is noted that the small impurity $\mathrm{PdSn}_{2}$ superconducts below $3.34 \mathrm{~K}$ [13] which is however somewhat above our observation of superconductivity in $\mathrm{Yb}_{3} \mathrm{Pd}_{4} \mathrm{Sn}_{13}$. Aside from the superconductivity features in the title compound, the electronic specific heat becomes strongly enhanced at low temperatures. It is very tempting to investigate $\mathrm{Yb}_{3} \mathrm{Pd}_{4} \mathrm{Sn}_{13}$ further to look for features of strongly correlated electrons with high effective masses. Quantitative studies await the procurement of single crystal sample material of suitable size for conducting physical properties measurements.

\section{Acknowledgments}

A.M.S. thanks the FRC/URC of UJ, and the SA NRF (93549) for financial assistance. R.F.D. thanks the Faculty of Science of UJ for a scholarship towards MSc studies.

\section{References}

[1] P.J. Webster, Contemp. Phys. 10, 559 (1969).

[2] M.R.R. Norman, J. Supercond. Nov. Magn. 25, 2131 (2012).

[3] G.S. Nolas, D.T. Morelli, T.M. Tritt, Annu. Rev. Mater. Sci. 29, 89 (1999).

[4] E. Schuberth, M. Tippmann, L. Steinke, S. Lausberg, A. Steppke, M. Brando, C. Krellner, C. Geibel, R. Yu, Q. Si, F. Steglich, Science 351, 6272 (2016).

[5] F. Gastaldo, M. Giovannini, A.M. Strydom, R.F. Djoumessi, I. Čurlík, M. Reiffers, P. Solokha, A. Saccone, J. Alloys Comp. 694, 185 (2017).

[6] T. Muramatsu, T. Kagayama, K. Shimizu, Y. Aoki, H. Sato, M. Giovannini, P. Bonville, V. Zlatic, I. Aviani, R. Khasanov, C. Rusu, A. Amato, K. Mydee, M. Nicklas, H. Michor, E. Bauer, Phys. Rev. B 83, 180404(R) (2011).

[7] A.M. Strydom, D. Britz, J. Low. Temp. Phys. 179, $62(2015)$.

[8] W. Kraus, G. Nolze, J. Appl. Crystallogr. 29, 301 (1996).

[9] S. Paschen, M. Baenitz, V.H. Tran, A. Rabis, F. Steglich, W. Carrillo-Cabrera, Y. Grin, A.M. Strydom, P. de V. du Plessis, J. Phys. Chem. Solids 63, 1183 (2002).

[10] A. Prokofiev, A. Sidorenko, K. Hradil, M. Ikeda, R. Svagera, M. Waas, H. Winkler, K. Neumaier, S. Paschen, Nat. Mater. 12, 1096 (2013).

[11] A.M. Strydom, J. Phys. Condens. Matter 19, 386205 (2007).

[12] O.V. Lounasmaa, Phys. Rev. B 129, 2460 (1963).

[13] L.I. Berger, B.W. Roberts, in: CRC Handbook of Chemistry and Physics, 91st ed., CRC Press, Boca Raton 2010-2011. 